\title{
The Maia Detector Journey: Development, Capabilities and Applications
}

C G Ryan ${ }^{1}$, D P Siddons ${ }^{2}$, R Kirkham ${ }^{1}$, A J Kuczewski ${ }^{2}$, P A Dunn ${ }^{1}$, G De Geronimo ${ }^{2}$, A. Dragone ${ }^{2}$, Z $\mathrm{Y} \mathrm{Li}^{2}$, G F Moorhead ${ }^{1}$, M Jensen ${ }^{1}$, D J Paterson ${ }^{3}$, M D de Jonge ${ }^{3}$, D L Howard ${ }^{3}$, R Dodanwela ${ }^{1}$, G A Carini $^{2}$, R Beuttenmuller ${ }^{2}$, D Pinelli ${ }^{2}$, L Fisher ${ }^{1}$, R M Hough ${ }^{1}$, A Pagès ${ }^{1}$, S A James ${ }^{3}$ and P Davey ${ }^{1}$

${ }^{1}$ CSIRO Mineral Resources, Gate 5 Normanby Rd., Clayton VIC 3168, Australia

${ }^{2}$ Brookhaven National Laboratory, Brookhaven, Upton NY, USA

${ }^{3}$ Australian Synchrotron, Blackburn Road, Clayton VIC 3168, Australia

The development of the Maia detector was motivated by the desire for high throughput synchrotron fluorescence element mapping with images beyond $100 \mathrm{M}$ pixels, which capture both fine detail and extended spatial context. It achieves this by using a large planar silicon detector array together with event-mode data acquisition, where each detected X-ray event is tagged with position in the scan, thus avoiding the readout overheads of a "step and acquire" style of mapping. The sample stage moves continuously and the stage encoders are read by the FPGA based real-time processor to provide coordinates for each event, which provides great freedom in scan speed or transit time per map pixel [1]. The large detector array, with 384 independent detector channels, each with its own charge amplification and pulse capture electronics, implemented using custom ASICs, enables cumulative count-rates exceeding $10 \mathrm{M} / \mathrm{s}$ to be achieved with low pile-up probability, which enables adequate counting statistics to be acquired per pixel in transit times as short as $50 \mu$ s [2,3]. The array uses a back-scatter, annular configuration, which combines a 1.3 sr solid-angle detection geometry with complete freedom in sample size and scanning range. This has enabled applications to extend from the initial focus on synchrotron X-ray microanalysis, with $\mu \mathrm{m}$ sized pixels and scan ranges up to a few centimeters, to macro-scale mapping using synchrotron or laboratory X-ray sources, with scan ranges up to $\sim 1 \mathrm{~m}$.

The Maia concept was introduced at M\&M 2005 and prototypes demonstrated performance using a 32 channel array at the NSLS synchrotron in 2006 [4] and a 96 channel array at the NSLS and Australian Synchrotron (AS) in 2008 [5,6]. The 96-channel prototype demonstrated imaging up to $77 \mathrm{M}$ pixels and transit times as short as $50 \mu \mathrm{s}$ and demonstrated real-time spectral deconvolution for quantitative imaging using the Dynamic Analysis algorithm implemented in the FPGA [5]. An annular 384 channel Maia detector was commissioned for the XFM beamline of the AS [2,3] and from 2011 it has demonstrated imaging beyond $100 \mathrm{M}$ pixels (largest image $1 \mathrm{G}$ pixels; Fig. 1) with more than $10^{5}$ images and $\sim 100 \mathrm{~TB}$ of event data acquired. Now two Maia 384 detectors are in routine application at the XFM beamline [7], one for $\mu \mathrm{m}$-scale mapping and one for macro-scale mapping of cultural heritage objects. Maia 384 detectors are also in use for SXRF element mapping at the P06 beamline, Petra III synchrotron, DESY in Hamburg [8], the SRX beamline, NSLS-II in New York and the CHESS synchrotron at Cornell University. The large solid-angle and collection efficiency of the Maia 384 detector has been exploited in a pair of laboratory XRF mapping systems called Maia Mapper at CSIRO for high definition element mapping of rock samples and drill core at $30 \mu \mathrm{m}$ spatial resolution over spatial scales up to $500 \mathrm{~mm}[9]$.

Synchrotron XRF mapping using Maia has been applied to studies in the earth, planetary, environmental, medical, biological, chemical and material sciences as well as cultural heritage (see refs cited in [1]). Methods and quantitative imaging techniques developed for Maia, which exploit event acquisition tagged by two or three axis coordinates, include large area, high definition, high throughput 
mapping of complex samples [1] (Fig. 1), depth contrast mapping and particle depth determination [10], 3D fluorescence tomography [11], XANES image stacks [12] and XANES slice tomography and quick XANES mapping where beam energy may be the fast axis [13] [14].

[1] C G Ryan et al., Journal of Physics: Conference Series 499 (2014) 012002.

[2] R Kirkham et al., AIP Conference series 1234 (2010) 240-243.

[3] D P Siddons et al., Journal of Physics: Conference Series 499 (2014) 012001.

[4] D P Siddons et al., 2006 IEEE Medical Imaging Conf. 2, 4179111, (2007) 725.

[5] C G Ryan et al., AIP Conference Proc. 1221 (2010) 9.

[6] C G Ryan et al., Nucl. Instr. Meth A 619 (2010) 37.

[7] D Paterson et al., AIP Conf. Proc. 1365 (2011) 219.

[8] U Boesenberg et al., J. Synchrotron Rad. 23 (2016) 1550.

[9] C G Ryan et al., J. Instrumentation, in press.

[10] C G Ryan et al., Proc. SPIE 8851, X-Ray Nanoimaging (2014) 88510Q.

[11] E Lombi et al., Analytical and Bioanalytical Chemistry 400 (2011) 1637.

[12] B Etschmann et al., Environmental Chemistry 11 (2014) 341.

[13] U Boesenberg et al., J. Synchrotron Rad., in press.

[14] Work performed at the XFM beamline of the Australian Synchrotron and supported by the CSIRO Science and Industry Endowment Fund.
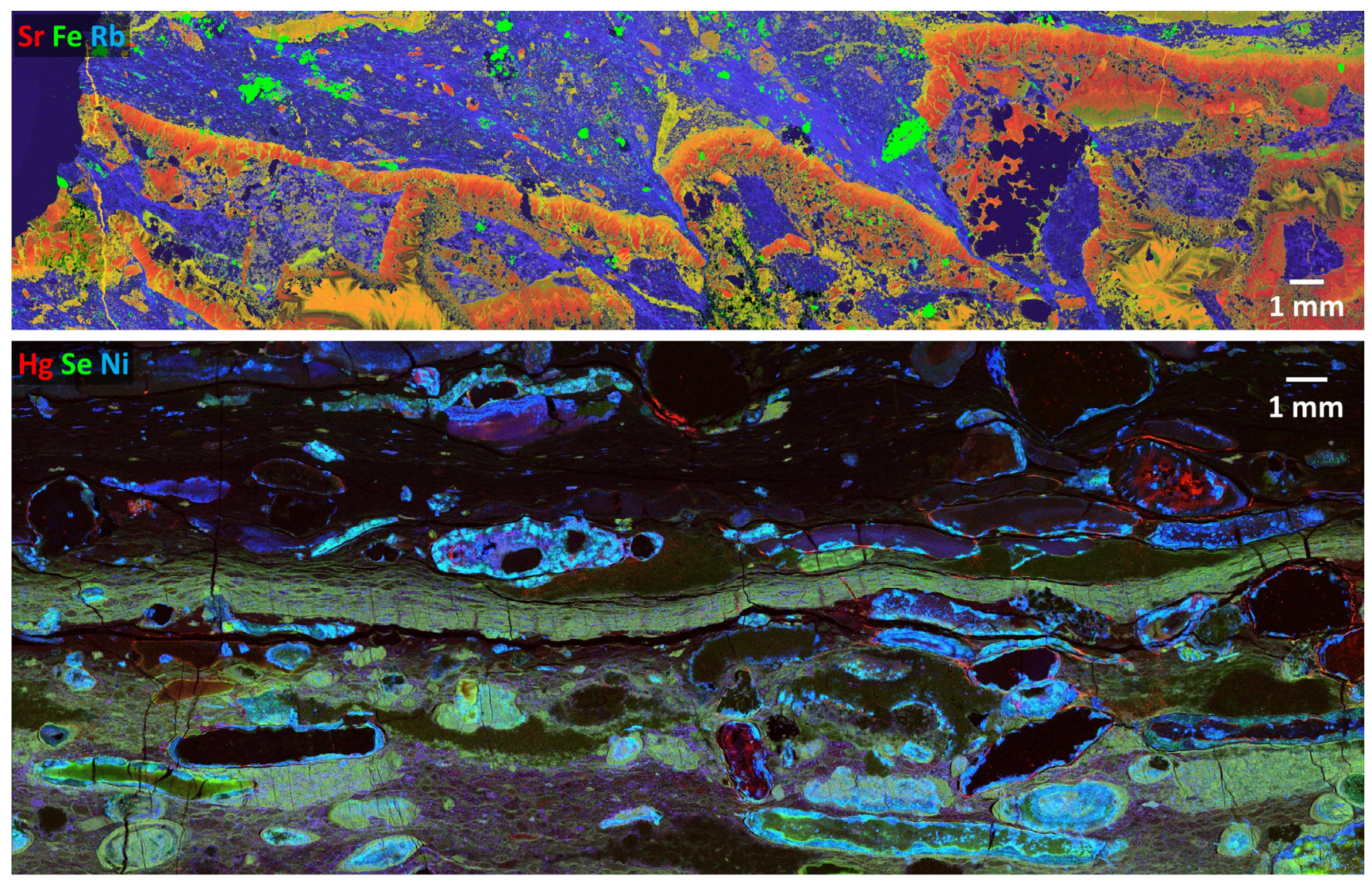

Figure 1. XFM Maia images (18.5 keV X-rays) of samples from the a) Sunrise Dam Gold Mine ( $\mathrm{Sr}, \mathrm{Fe}$ and $\mathrm{Rb}$ in RGB, $66668 \times 15001$ pixels, $40.0 \times 9.0 \mathrm{~mm}^{2}, 0.13 \mathrm{~ms} /$ pixel, $1 \mathrm{G}$ pixels), b) Nick metalliferous shale (Hg, Se and Ni in RGB, 8276 x 4526 pixels, 33.1 x $18.1 \mathrm{~mm}^{2}, 0.67 \mathrm{~ms} /$ pixel, 37M pixels). 